\title{
Synchrotron Oscillations Effects on Observations of an RF-solenoid Spin Resonance for a Polarized Deuteron Beam at COSY
}

\author{
Greta Guidoboni* \\ University of Ferrara and INFN Ferrara \\ 44100 Ferrara, Italy \\ E-mail: guidoboni@fe.infn.it
}

\section{Edward J. Stephenson}

Center for Exploration of Energy and Matter

Indiana University, 2401 Milo. B. Sampson Lane, Bloomington, IN 47408 USA

E-mail: stepheneeindiana.edu

\section{Paolo Lenisa}

University of Ferrara and INFN Ferrara

44100 Ferrara, Italy

E-mail: lenisa@fe.infn.it

\begin{abstract}
The search for an electric dipole moment (EDM) using a polarized, charged-particle beam in a storage ring requires ring conditions that can maintain a longitudinal, and stable, polarization for times up to $1000 \mathrm{~s}$. The EDM signal is a rotation of this polarization into the vertical direction as a consequence of the radial electric fields present in both electric and magnetic storage rings. A study is beginning at the COoler SYnchrotron (COSY) located at the Forschungszentrum-Jülich to examine the effects of emittance and momentum spread on the spin coherence time. As these effects also appear in the properties of an RF-induced spin resonance, this study began by exciting the $1-G \gamma$ resonance with fixed and variable frequency RF-solenoid scans and a $0.97 \mathrm{GeV} / \mathrm{c}$ polarized deuteron beam. Subsequent model analysis of the data recorded for many different beam and solenoid conditions demonstrated through good agreement with the data that most of the observed effects originated in the time shift of deuterons passing through the solenoid, as they underwent synchrotron oscillations inside first harmonic beam bunching. A model, which does not include the ring lattice, is described along with a summary of phenomena observed during the experiment.
\end{abstract}

8th International Conference on Nuclear Physics at Storage Rings-Stori11,

October 9-14, 2011

Laboratori Nazionali di Frascati dell'INFN, Italy

\footnotetext{
* Speaker.
} 


\section{Introduction}

The Electric Dipole Moment (EDM) is a permament charge separation within the particle volume, aligned along the spin axis. The EDM violates both parity conservation and time reversal invariance. Under the CPT theorem, it violates CP asymmetry and could indicate the reason why the universe is dominated by matter. The Standard Model (SM) predicts a non-vanishing but unobservably small $\operatorname{EDM}\left(e . g\right.$., for proton $\left|d_{p}\right|_{S M}<10^{-32} \mathrm{e} \cdot \mathrm{cm}$ ) that is too small to explain the baryon asymmetry. Models beyond the SM predict values within the sensitivity reach of current or planned experiments (for proton and deuteron $\left|d_{p, d}\right| \rightarrow 10^{-29} \mathrm{e} \cdot \mathrm{cm}$ ), so that EDM searches represent a sensistive probe for new physics. At the moment, no EDM has been observed on a fundamental particle.

Detection methods rest mainly on observing the precession of the polarization in an external electric field. Beginning with a longitudinally polarized beam in a storage ring, the EDM can be detected as a rotation of the polarization from the longitudinal to the vertical direction, due to the interaction with the inward radial electric field that is always present in the particle frame. In a normal storage ring, the polarization will precess about the vertical direction, giving an anomalous rotation relative to the velocity of:

$$
\vec{\omega}_{a}=\vec{\omega}_{s}-\vec{\omega}_{c}=-\frac{q}{m}\left\{a \vec{B}+\left[a-\left(\frac{m}{p}\right)^{2}\right] \frac{\vec{\beta} x \vec{E}}{c}\right\}
$$

where $\omega_{s}$ is the spin precession in the horizontal plane, $\omega_{c}$ the particle angular frequency and $a=\frac{g-2}{2}$ the anomalous moment. In order to keep the spin aligned along the velocity, the frozen spin technique requires that $\omega_{a}$ vanish. Since the proton $a=1.79$ and deuteron $a=-0.14$ have anomalous moments of the opposite sign, this requirement can be satisfied in two different ways. In the proton case at $p=0.7 \mathrm{GeV} / \mathrm{c}$ ("magic momentum") the expression in square brackets is zero. In a pure electric ring the magnetic field is absent and $\omega_{a}$ is also zero. A magic momentum for deuterons does not exist. Using both magnetic and outward electric fields, the frozen spin condition is fulfilled. In order to achieve a sensitivity of $10^{-29} \mathrm{e} \cdot \mathrm{cm}$, a good compromise for the experiment requires a polarimeter sensitivity of $10^{-6} \mathrm{rad}$ and a storage time of $1000 \mathrm{~s}$, during which the spin decoherence has to be minimized.

\section{Beam polarization measurements at COSY}

Development of the techniques needed for the deuteron EDM experiment has started at COSY (COoler SYnchrotron) in Jülich, Germany. The goal of the January 2011 beamtime was the investigation of the effects of beam momentum spread and emittance on the spin coherence time. It was discovered instead that synchrotron oscillation effects dominated the measurements. This paper describes these effects.

As momentum spread and emittance effects also appear in the properties of an RF-induced spin resonance, this study was designed to investigate first the $1-G \gamma$ resonance of a polarized deuteron beam. The EDDA scintillator detectors were used as a mock EDM polarimeter to measure the vertical component of the polarization. The beam was moved slowly toward a $15 \mathrm{~mm}$ thick carbon target located along the side of the beam to provide a continuous beam extraction during the store. 


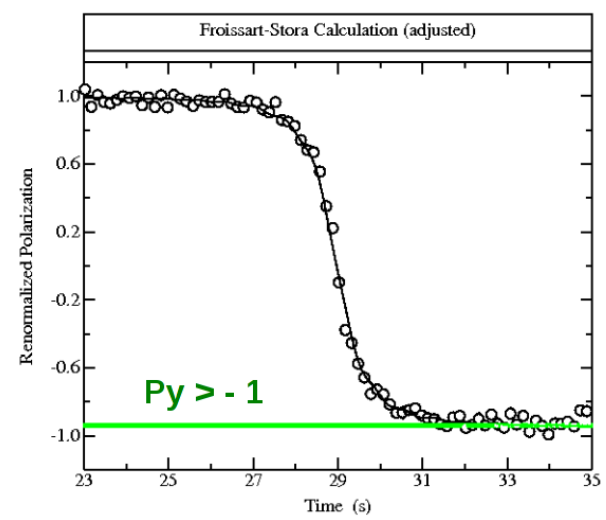

(a) Froissart-Stora scan, uncooled beam

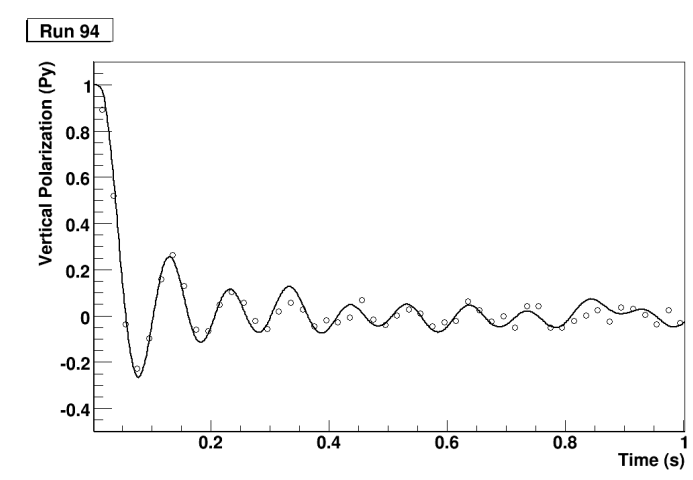

(b) Fixed frequency scan, uncooled beam

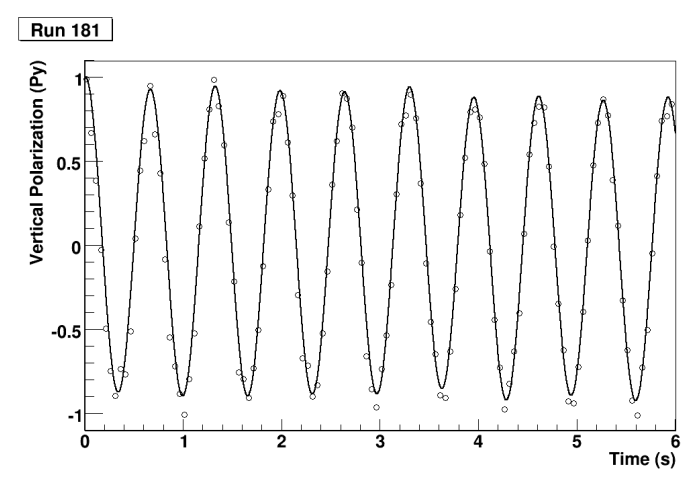

(c) Fixed frequency scan, cooled beam

Figure 1: Beam polarization measurements at COSY in 2011.

Elastic deuterons were detected since elastic scattering is a spin sensitive process and the cross section for $\mathrm{d}+\mathrm{C}$ scattering is large. More details on the polarimeter studies can be found in $[1,2]$.

The measurements in 2011 were performed with a polarized deuteron beam of $p=0.97 \mathrm{GeV} / \mathrm{c}$ circulating in COSY with a cyclotron frequency of $f_{c y c}=750602.5 \pm 0.5 \mathrm{~Hz}$ and a relativistic factor $\gamma=1.12583$. The synchrotron oscillation frequency was $f_{\text {syn }}=331 \mathrm{~Hz}$ and the maximum RF-solenoid strength $\varepsilon=2.66 \cdot 10^{-5}$ rev. The beam was bunched at the first harmonic $(\mathrm{h}=1)$. Polarization data were taken with uncooled and electron-cooled beam.

The first measurement was a Froissart-Stora frequency scan with an uncooled beam to identify the spin resonance frequency, given by $f_{\text {res }}=f_{c y c}(1-G \gamma)$. The scan started $200 \mathrm{~Hz}$ below the resonance frequency and stopped $200 \mathrm{~Hz}$ above with a linear ramping of $10 \mathrm{~Hz} / \mathrm{s}$. When the initially vertically polarized beam crosses the resonance, a polarization flip is observed. As will be discussed later, synchrotron oscillation effects prevented the flip from being complete (see Figure 1(a)). After that, a series of fixed frequency measurements of the time evolution of the polarization with the RF-solenoin tuned on the resonance frequency were expected to yield information on the RF-solenoid resonance width, which depends on momentum spread and emittance of the beam (see Figures 1(b) and 1(c)). In practice, the comparison between cooled and uncooled beams was complicated by the effects of synchrotron oscillation amplitudes. To describe the data, a so-called "no lattice model" including synchrotron oscillations has been developed. 


\section{No lattice model}

The "no lattice model" is based on a very simple idea. It does not provide particle tracking because the lattice is not included. The storage ring is represented by a circumference the length of COSY with a constant vertical magnetic field. The spin orientation of each particle is changed at the end of each turn by two 3-dimensional rotation matrices. One represents the spin precession in the bending magnets and rotates the spin vector around the vertical axis $(\hat{y})$. The rotation angle is related to the spin tune $v_{s}=G \gamma$ and thus to the particle velocity. The second one reproduces the RF-solenoid effect as a small spin rotation around the longitudinal axis $(\hat{z})$. The rotation angle depends on the solenoid strength and the time when particle passes through the RF-solenoid.

Synchrotron oscillations are added to the model using simple harmonic motion. A sinusoidal function (see Equation. 3.1) describes the particle position along the bunch as a function of time:

$$
z(t)=A \cdot \sin \left(2 \pi f_{\text {sync }} t+\Phi_{\text {sync }}\right)
$$

where $A$ is the synchrotron oscillation amplitude. The distribution of $A$ values reflects the momentum spread. If the particle is at the endpoint of the bunch, $\Delta p / p=0$. When it crosses the center of the bunch, the momentum spread is maximum. Thus, synchrotron oscillations affect the spin tune, which depends on particle velocity, and the transit time through the RF-solenoid. From the model, results for the vertical spin component as a function of time are plotted for particles undergoing different synchrotron amplitudes in Figure 2. All the sinusoidal functions oscillate between 1 and -1 but with different frequencies: the larger is the amplitude, the slower is the frequency.

In order to reproduce the data of Figures 1(b) and 1(c), the vertical beam polarization $P_{y}$ has been calculated as a linear combination of single-particle functions $f\left(A_{i}, t\right)$ :

$$
P_{y}(t)=a_{1} f\left(A_{1}, t\right)+\ldots+a_{N} f\left(A_{N}, t\right)
$$

where the $a_{i}$ coefficients represent the weight of the related amplitude to the total polarization. The next section will show the importance of determining the proper amplitude distribution to reproduce the data.

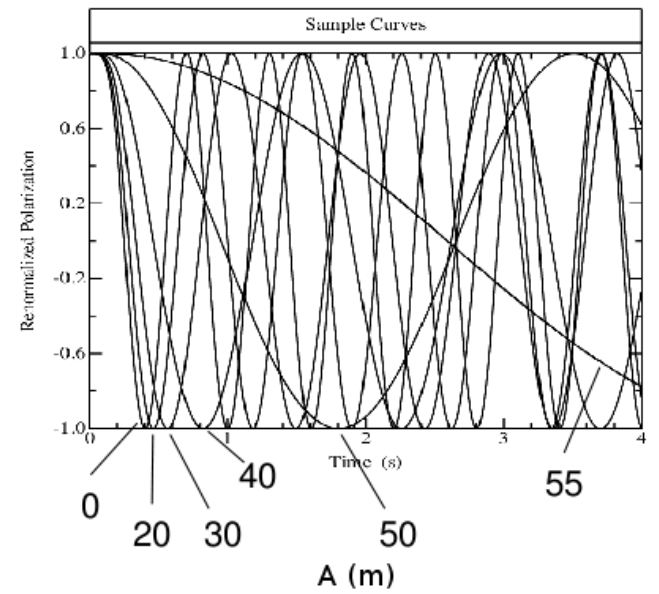

Figure 2: Each curve is a function $f\left(A_{i}, t\right)$ representing the vertical spin component as a function of time for a particle undergoing a synchrotron oscillation of amplitude $A$.

\section{Data Analysis}

The "no lattice model" has been applied to three different data samples. The data have been normalized to one for the time before the RF-solenoid is energized. The time dependence afterward is reproduced by choosing a set of amplitudes with $i=1, N$ in Equation 3.2 and adjusting the $a_{i}$ to reproduce the data. Starting from an on-resonance fixed frequency scan of a cooled beam, the recorded data are presented in Figure 1(c), where the vertical polarization oscillates about zero 


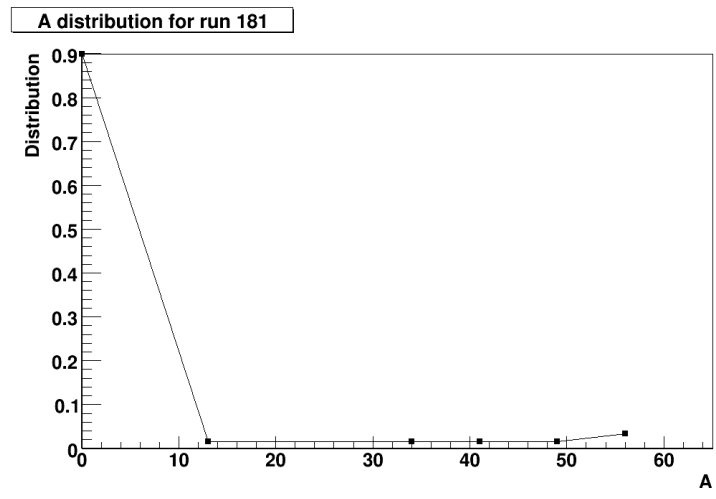

(a) Amplitude distribution for a cooled beam.

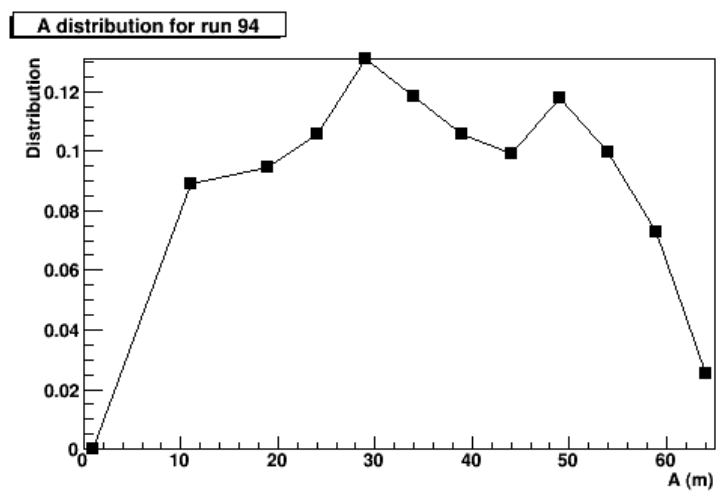

(c) Amplitude distribution for an uncooled beam.

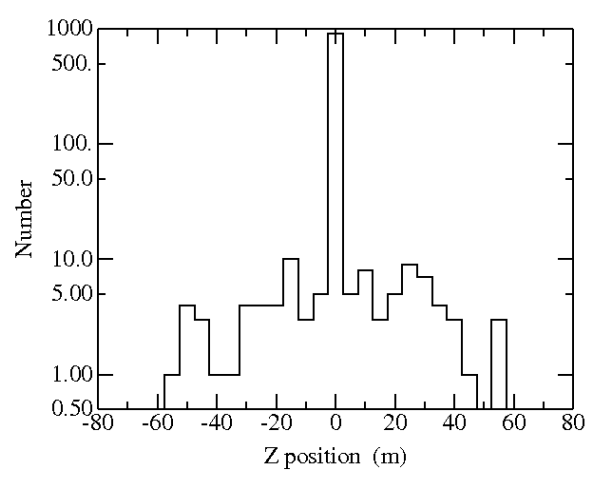

(b) Distribution of 1000 particles in the bunch of a cooled beam.

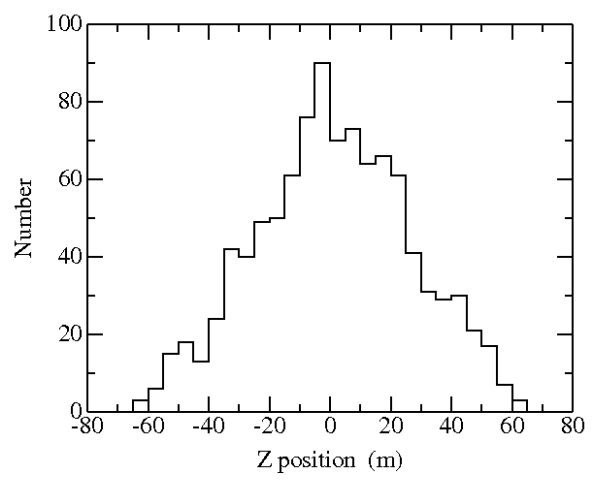

(d) Distribution of 1000 particles in the bunch of an uncooled beam.

Figure 3: Analysis for the fixed frequency measurment with a cooled and uncooled beam.

with an amplitude less than one. The explanation of this behavior can be found in Figure 3(a): there is a non-zero contribution from high values of the oscillation amplitude coming from the uncooled part of the beam. As shown in Figure 3(b), 91\% of the particles are concentrated in the center of the beam while $9 \%$ are spread out, causing the depolarization. Nevertheless, the experimentally observed oscillation frequency is the same as for the reference particle that does not undergo synchrotron oscillations. The data shown in Figure 1(b) where damped oscillations characterize the time dependence of the polarization are from the uncooled beam. The amplitude distribution is shown in Figure 3(c). In order to reproduce the damped oscillation it is important to know in detail the shape of the amplitude distribution curve. Figure 3(d) shows that the particles are distributed along the whole bunch and cross the RF-solenoid at different times. The RF-solenoid effect will be stronger for particles with a small synchrotron oscillation amplitude and weaker in the other case as reflected by the slower polarization oscillation from the model. Since the beam polarization is an avarage on over all particle spins, the result is a damped oscillation. In both the cooled and uncooled cases, the time dependence of the polarization produced by the particle distributions shown in Figure 3 generate the time-dependent curves shown in Figures 1(b) and 1(c).

A different study of synchrotron oscillation effects on the RF-solenoid efficency has been developed with the analysis of the Froissart-Stora scan data for an uncooled beam. 
Data are reported in Figure 1(a), where a non-complete polarization flip is visible. In order to determine the contribution of each amplitude, a FSscan simulation has been performed for each single-particle function, depending on a different $A$ value. In Figure 4 , the final vertical spin component is plotted against the corresponding A value. For amplitudes $\leq 48 \mathrm{~m}$ the spin flip is complete. Then the weakening of the RF-solenoid effect results in incomplete spin flip until $A=$ $58 \mathrm{~m}$ where there is no spin flip at all. For larger amplitudes the spin-filp resumes. The conclusion is that the in-

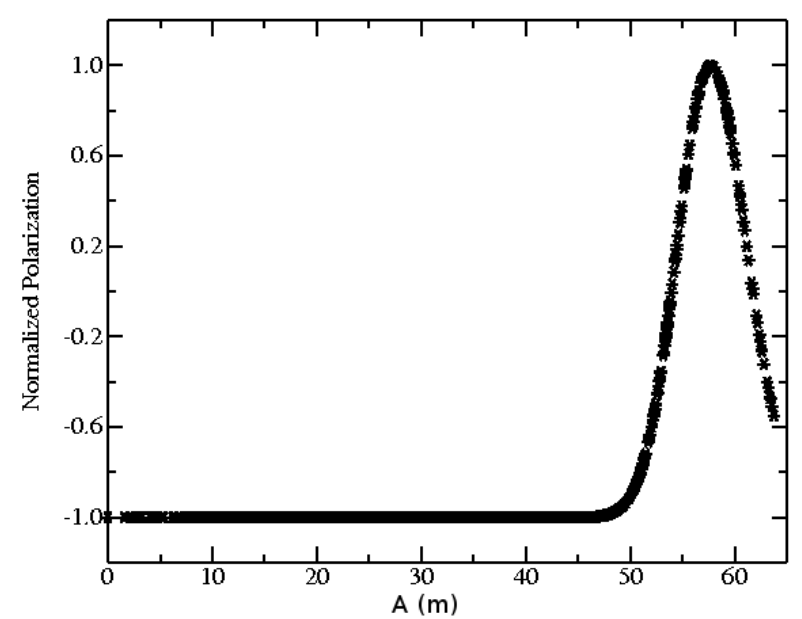

Figure 4: The final vertical spin component of a singleparticle function is plotted against the corresponding synchrotron oscillation amplitude $A$. For $A \leq 48 \mathrm{~m}$ the spin flip is complete, the RF-solenoid starts being less efficient until when $A=58 \mathrm{~m}$ where there is no spin flip at all. complete polarization flip in the data of 1(a) represents the contribution of synchrotron oscillation amplitudes beyond $48 \mathrm{~m}$.

\section{Conclusions}

A "no lattice model" that includes synchrotron oscillation effects has been developed to study the spin coherence time in a storage ring. The model has been successfully applied to reproduce polarization data aquired at the COSY storage ring. Fixed frequency scans shown that finding the correct synchrotron amplitude distribution is fundamental to reproduce the data. The uncooled beam wiggles are important to define the details of this distribution in a manner analogous to a Fourier transform. Froissart-Stora scans demonstrated how the RF-solenoid flip efficency can be altered by the synchrotron oscillations, due to the change in the particle transit time through the solenoid. In particular, for large amplitudes the RF-solenoid can no longer completely flip the spin.

The experimental apparatus used for these measurements was not sensitive to the horizontal polarization component. A new data acquisition system forseen for the coming year will be built to record a time stamp for each event. Unfolding the spin precession will give a direct measurement of the horizontal polarization and the spin coherence time, opening the possibility of a new way of observing the effects of changing emittance and momentum spread.

The authors acknowledge financial support from the Italian INFN, the Helmholtz Foundation, the EU and the US NSF and DOE.

\section{References}

[1] N. P. M. Brantjes et al., Correction systematic errors in high-sensitivity deuteron polarization measurements. NIM A 664 (2011) 49.

[2] E. J. Stephenson, Polarimeter and Spin Coherence Time Developments at COSY for a Storage Ring EDM search, in proceedings of STORI'11 conference, PoS(STORI11)009. 